\title{
Visual globes, celestial spheres, and the perception of straight and parallel lines
}

\section{Brian Rogers, Cassandra Rogers}

Department of Experimental Psychology, University of Oxford, South Parks Road, Oxford OX1 3UD, UK: e-mail: bjr@psy.ox.ac.uk

Received 14 November 2008, in revised form 28 May 2009

\begin{abstract}
Helmholtz's famous distorted chessboard pattern has been used to make the point that perception of the straightness of peripherally viewed lines is not always veridical. Helmholtz showed that the curved lines of his chessboard pattern appear to be straight when viewed from a critical distance and he argued that, at this distance, the contours stimulated particular 'direction circles' in the field of fixation. We measured the magnitude of the distortion of peripherally viewed contours, and found that the straightness of elongated contours is indeed misperceived in the direction reported by Helmholtz, but that the magnitude of the effect varies with viewing conditions. On the basis of theoretical considerations, we conclude that there cannot, in principle, be particular retinal loci ('loci' is used here in the sense of an arc or an extended set of points that provide a basis for judging collinearity) to underpin our judgments of the straightness and parallelity of peripheral contours, because such judgments also require information about the 3-D surface upon which the contours are located. Moreover, we show experimentally that the contours in the real world that are judged to be straight and parallel can stimulate quite different retinal loci, depending on the shape of the 3-D surface upon which they are drawn.
\end{abstract}

\section{Introduction}

In the 1st edition of the Handbuch der physiologischen Optik, Helmholtz asked a deceptively simple question: "Welche sind die ungekrümmte ... Linien im Sehfelde?" "Which are the uncurved lines on the visual globe?" (Helmholtz 1867, 1910/1962, page 178). (1) To answer this question, we first need to consider the concept of the 'visual globe'. For Helmholtz, the 'visual globe' (Sehfeld) is a sphere, with the observer's eye at its centre, that moves with the eye and is to be distinguished from the field of fixation or 'celestial sphere' (Blickfeld) that remains fixed with respect to the head (see also Rogers and Brecher 2007). Eye movements are used to move the visual globe and thereby sample the celestial sphere, and the magnitude and direction of those movements may be visualised by projecting the fixation line of the visual globe onto the surface of the celestial sphere. Helmholtz wrote:

"The latter [visual globe] is regarded as moving with the eye so that the image of every point on it remains constantly at the same definite place on the retina." (Helmholtz $1910 / 1962$, page 164)

However, if there is a direct mapping between the visual globe and the retinal image, the question arises why Helmholtz preferred to talk about the visual globe and the celestial sphere rather than the retina (which moves with the eye) and the retinal image (which does not)? Helmholtz answered:

"I prefer to consider the two surfaces [the visual globe and the celestial sphere] that are outside the eye rather than the retina and the retinal image, because the former are a more correct expression of our actual consciousness, and because by directly referring all

(1) One of the reviewers raised the question whether Southall's translation of 'Sehfeld' as 'visual globe' (rather than 'visual field') was correct. Helmholtz (1910/1962), however, wrote that 'Sehfeld' was best considered as a "spherical shell concentric with the field of fixation" (page 165) and hence visual globe does indeed seem to be the more appropriate term. 
places to the two spherical fields we avoid the ambiguity that is responsible for so much that is erroneous here; whereas when we speak of knowing the positions of objects by the places on the retina that are affected by them, we seem to imply that we are aware of the retina and know something about its dimensions and extent. ... Moreover, so far as vision is concerned, I myself am disposed to think that neither the size, form and position of the real retina nor the distortions of the image projected on it matter at all, as long as the image is sharply delineated all over ..." (Helmholtz 1910/1962, page 166)

Helmholtz's justification for introducing the concepts of the visual globe and the celestial sphere can be seen as similar to the case made by Gibson, a hundred years later, in support of his idea of the 'optic array' (Gibson 1966). So what did Helmholtz mean by the "uncurved lines on the visual globe"? It might be thought that the question of the 'uncurved lines' could be answered by considering the underlying geometry - straight lines in the world project to great circles (or lines of longitude) on a spherical projection surface, like Helmholtz's celestial sphere (Howard and Rogers 1995; figure 1 here).

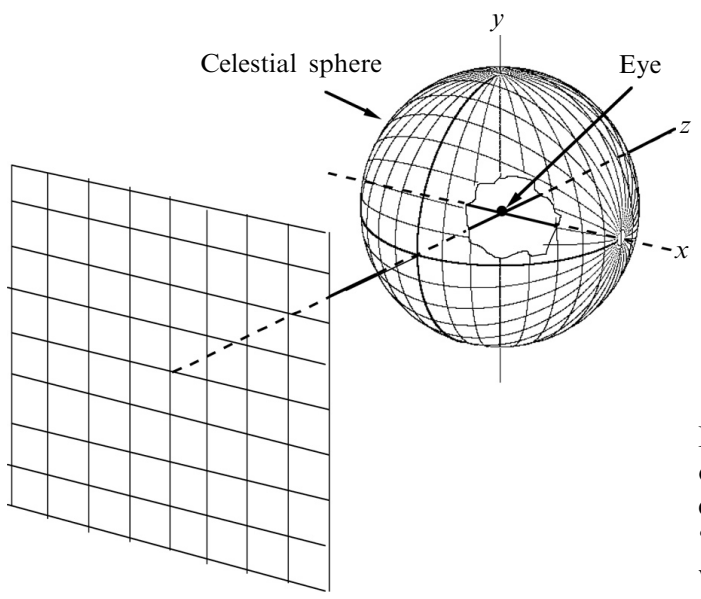

Figure 1. Vertical and horizontal straight lines drawn on a frontal surface (left) project to great circles or lines of longitude on Helmholtz's 'celestial sphere' - a spherical projection surface with the eye at its centre.

As a consequence, the 'uncurved lines' on the celestial sphere should consist of all the possible great circles covering the sphere. ${ }^{(2)}$ Moreover, any visual system could detect and register straight lines through the use of elongated receptive fields corresponding to all the possible arcs of these great circles (figure 1). However, it should be noted in passing that Helmholtz asked about the uncurved lines on the visual globe and these do not correspond to the uncurved lines on celestial sphere, except when the eyes are in the straight-ahead position, a point that we shall return to later. Notwithstanding this issue, it is important to realise that Helmholtz's question was not about geometry, but rather about perception and hence his question is perhaps better interpreted as: "Which lines projected on the surface of the visual globe are seen as straight?". Helmholtz wrote:

"If the field of fixation [celestial sphere] is imagined as being a spherical surface with its centre at the centre of rotation of the eye ... we might suppose that objective lines which were really straight lines in the external world, ... would be projected on the spherical field of fixation as arcs of great circles, would necessarily appear in the field of view as lines without curvature. But this is not so, except under certain conditions." (Helmholtz, 1910/1962, page 172) 
To summarise, the mathematically correct strategy for determining whether a line in space is straight would be to determine whether the projection of that line onto an idealised (spherical) retinal surface corresponded to a great circle. However, Helmholtz's observations show that this does not seem to be the case. In support of his claim, Helmholtz describes an experiment in which an observer is asked to judge whether three stars in the sky, that are collinear, appear to be aligned when the point of fixation is shifted some distance above or below the row of stars (figure 2). Under these conditions, Helmholtz noted that the three stars no longer appear to be aligned but instead appeared to lie on a line that is concave with respect to the fixation point. Helmholtz wrote:

"This shows that, when the eye looks steadily in one direction, a great circle of the celestial sphere will not appear to be without curvature unless it passes through the point of fixation; otherwise, it will appear concave towards that point." (Helmholtz, 1910/1962, page 179)

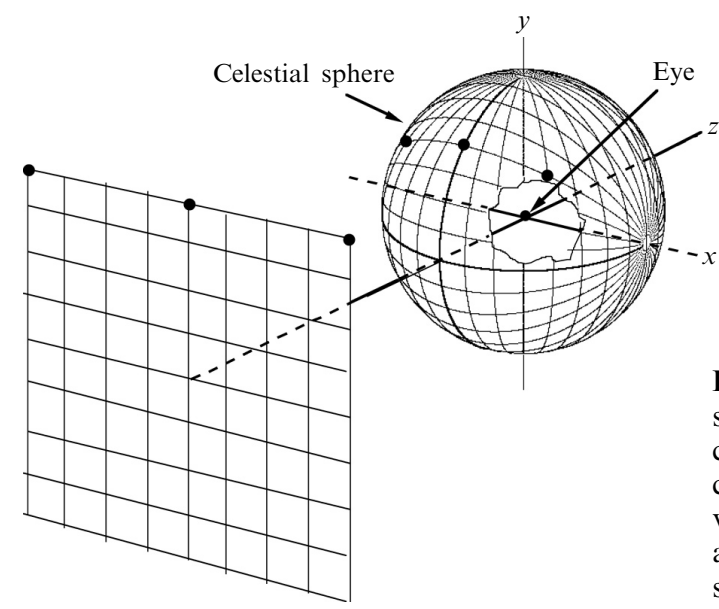

Figure 2. Helmholtz noted that three aligned stars (the dots) that project to the same great circle on a spherical projection surface - the celestial sphere - appear to be aligned when viewed directly, but no longer appear to be aligned when gaze is in a different direction, such as the straight-ahead.

If great circles do not represent the 'uncurved lines', which loci do? ${ }^{(3)}$ A second possibility is that the uncurved lines are the lines of latitude running parallel to the great circles passing through the point of fixation (figure 3). This figure shows that the lines on a frontal surface that project to horizontal and vertical lines of latitude on a spherical projection surface are all hyperbolae with their curvatures convex to the point of fixation (figure $3 a$ ).

Alternatively, figure $3 \mathrm{~b}$ shows that the parallel (and equally elevated) lines on a cylindrical, equidistance surface also map to lines of latitude. To test the hypothesis that the uncurved lines on the visual globe are lines of latitude, Helmholtz suggested the following:

"A strip of paper from three to five inches wide may be bent into the form of a semicylinder, and the eye placed on its axis. In looking at the middle of the strip, it seems to get wider toward the two ends and be bounded by two arcs with their convexities turned toward each other." (Helmholtz 1910/1962, page 180)

To summarise, lines (or points) in the world that project onto great circles are seen as concave towards the fixation point (figure 4a), and lines that project onto lines of latitude are seen as convex toward the fixation point (figure $4 \mathrm{~b}$ ). It follows that the lines that are seen as straight-the 'uncurved lines'-must correspond to some compromise loci between those two possibilities. 

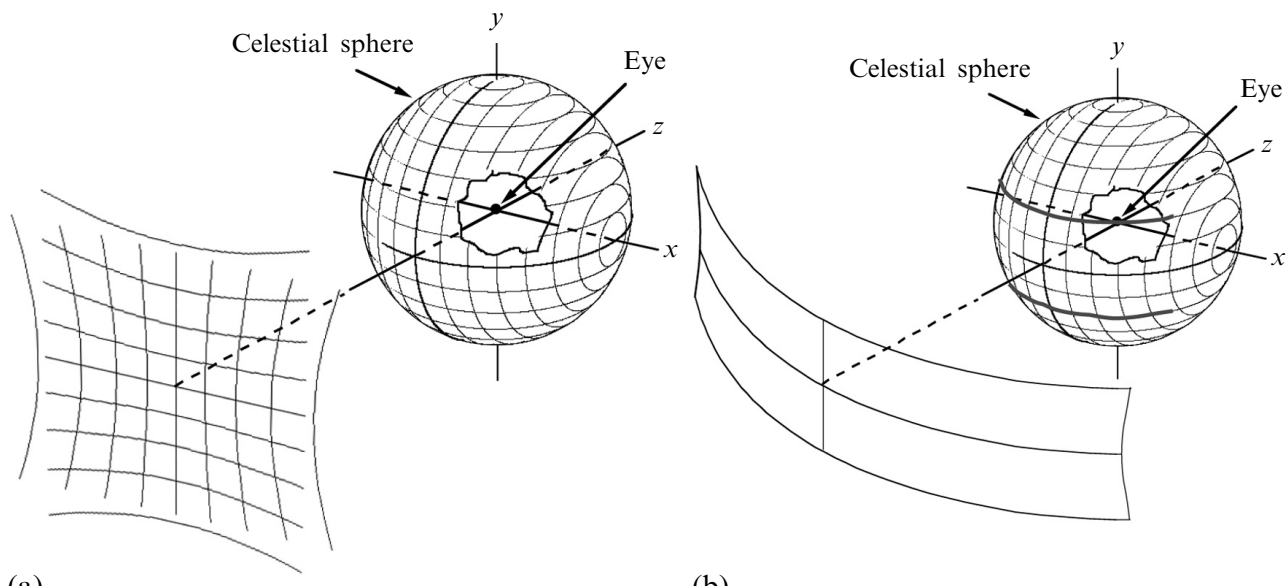

(a)

(b)

Figure 3. (a) The contours on a frontal surface that project to lines of latitude on a spherical projection surface are all hyperbolae. (b) But note that parallel lines on an equidistance surface also project to lines of latitude.
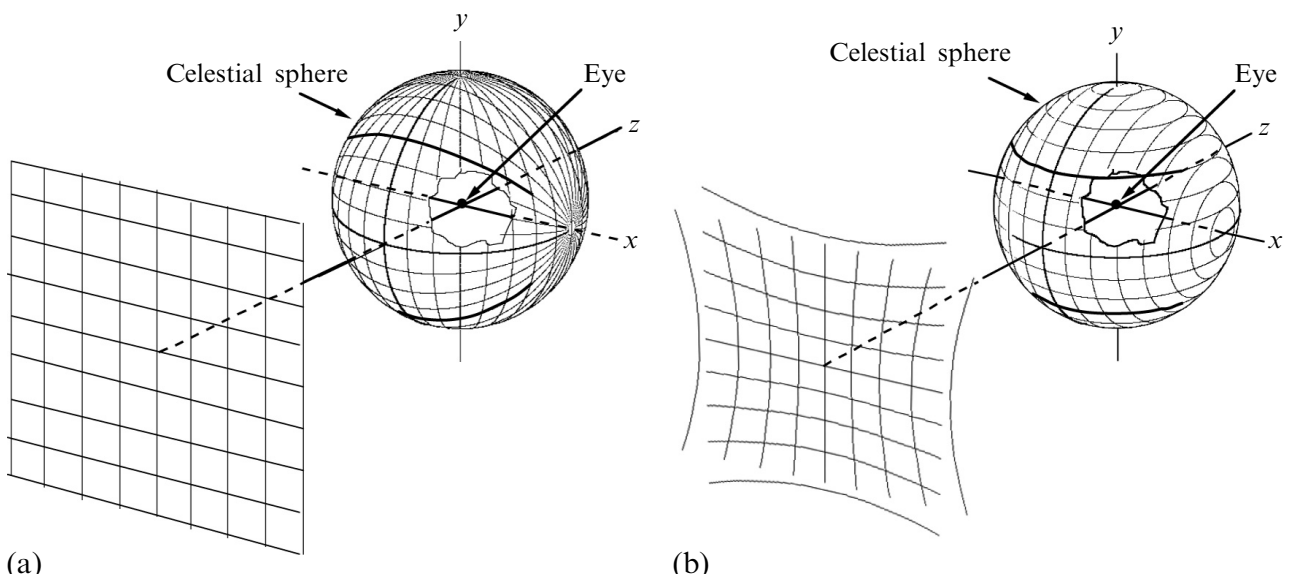

(b)

Figure 4. Given that straight and parallel contours (that project to great circles) are seen as curving concave towards the fixation point (a) and hyperbolae (that project to lines of latitude) are seen as curving convex (b), it follows that the lines on a frontal surface that are seen as straight must lie between these two possibilities.

The compromise loci that Helmholtz's chose are what he referred to as 'direction circles', which he defined in the following way:

"the circular arcs which are described by the line of fixation in turning around a fixed axis according to Listing's law." (Helmholtz 1910/1962, page 79)

When the eye is fixated in the straight-ahead or primary position, a subsequent eye movement will result in the line of fixation tracing out a great circle in the circular field of fixation (figure 4a). However, Helmholtz showed (figure 10 in Helmholtz 1910/1962) that, when the eye is fixated anywhere else in the visual field (ie in a secondary or tertiary position), the line of fixation during a subsequent eye movement "will not describe a great circle, but a circle parallel to the great circle corresponding to the atropic line belonging to its initial position" (as long as the eye movement obeys Listing's law). "These circles on the spherical field of fixation that pass through the occipital point will be called direction-circles". The occipital point is the point 
on the field of fixation exactly opposite the primary position of the line of fixation. Figure $5 \mathrm{~b}$ shows the locations of just six of the infinite number of possible direction circles. They can be thought of as being differently elevated circles from the horizontal and vertical equatorial planes of the celestial sphere. Figure 7 in Simonsz and Den Tonkelaar's (1990) article shows a similar depiction of a family of direction circles. They also make the point that a direction-circle coordinate system is preferable to other systems because the horizontal and vertical direction circles always intersect at $90^{\circ}$, as can be seen in figure $5 \mathrm{~b}$.

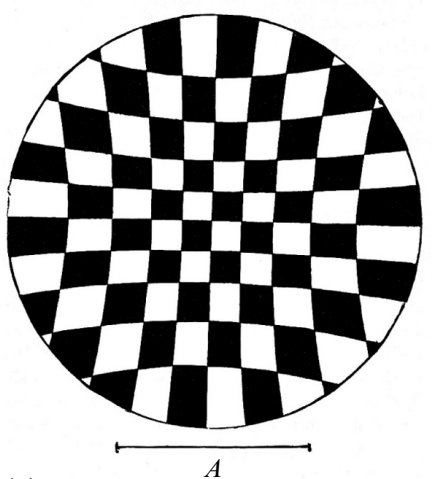

(a)

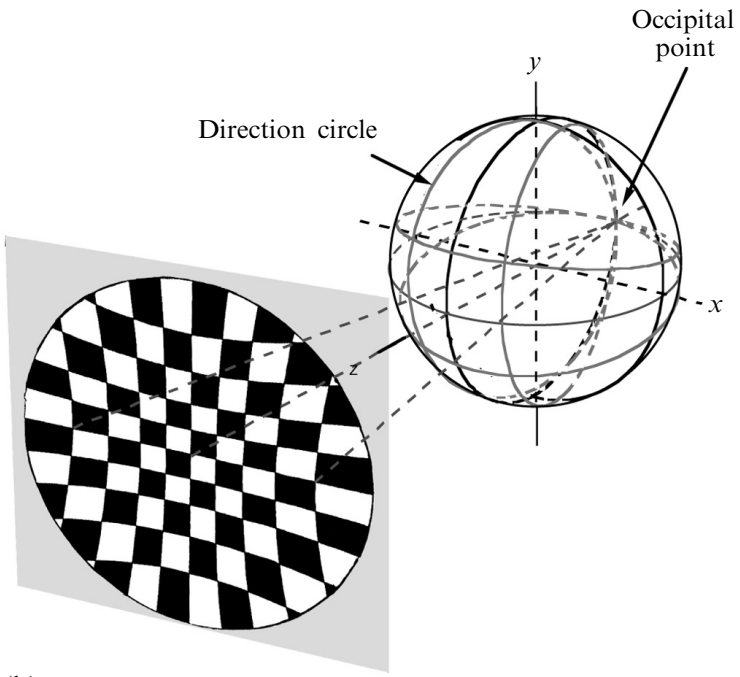

(b)

Figure 5. Helmholtz's distorted chessboard pattern (a) was designed so that the contours between the black and white checks project to particular 'direction circles' at the critical viewing distance $[A]$ indicated by the line underneath. All direction circles pass through the 'occipital point' on the celestial sphere located opposite to the principal line of fixation (b). Just six direction circles (three close to the horizontal meridian and three close to the vertical meridian) are shown here for clarity.

By comparing the locations of the direction circles to the locations of the great circles or the lines of latitude shown in figure 4, it is easy to see how they represent a compromise between these two possibilities. In particular, it can be seen that the elevation of a given direction circle at $90^{\circ}$ azimuth (to one side) is exactly halfway between the elevation of a corresponding line of latitude and the zero elevation of a corresponding great circle, as Helmholtz himself noted (1910/1962, page 183).

In order to test the hypothesis that the 'uncurved lines' on the celestial sphere correspond to his direction circles, Helmholtz constructed his famous distorted chessboard figure (figure 5a). He wrote:

\footnotetext{
"Accordingly, I have made a plane chart showing the projections of the direction-circles in the field of fixation which have the same directions as the vertical and horizontal lines going through the point of fixation"... The line $A$, reduced to the same scale indicates the distance of the observer's eye, which must be placed directly opposite the centre of the chart. ... under these circumstances, the direction circles in the field of fixation projected as hyperbolas, will appear as straight lines ...” (Helmholtz 1910/1962, pages $180-181$ )
}

The reader can verify for himself or herself whether the curved lines of the distorted chessboard pattern are seen as completely straight and parallel at the correct viewing distance, but Helmholtz's observations make it clear that straight lines in the world are typically not seen as straight when viewed in peripheral vision and, 
conversely, that the 'uncurved lines' on the field of fixation (celestial sphere), that are seen as straight, correspond to lines in the world that are actually curved.

Although the chessboard figure itself has been discussed extensively (Pirenne 1970; Hansen 1973; Anstis 1998; Liu and Schor 1998), there do not appear to be any studies that have tried to measure, in a more systematic way, the extent to which peripherally viewed lines have to be curved in order to be perceived as straight, apart from Parlee (1972) and Oomes et al (2008). The purpose of Parlee's study was to contrast the extent of misalignment of a pair of peripheral vertical lines, $10 \mathrm{deg}$ to the left or the right of the fixation point, with the misalignment of a peripherally located trio of three dots. Their results showed that, in order for the trio of three dots to appear to be aligned, they had to have a convex, chevron-like distortion between the centre and upper pair of dots and the centre and lower pair of dots of $\sim 5 \mathrm{deg}$. This finding is consistent in direction with the original Helmholtz chessboard illusion. However, Parlee found that there was little, if any, distortion when the three dots were replaced by a pair of lines forming a chevron-shaped figure. As the authors point out, their failure to find a distortion similar to Helmholtz's effect may have been due to the fact that observers could detect the abrupt change of orientation at the apex of the chevron. Hence, their finding does not have a direct bearing on our understanding of Helmholtz's chessboard illusion.

In a much earlier study, Küster (1876) found that an eccentrically located straight line, that would project to a great circle on a spherical projection surface (figure 2), was perceived as curved and, conversely, that a line with the curvature of one of Helmholtz's direction circles was perceived as straight. There was a crucial difference, however. The curvature or otherwise of Küster's lines (generated by an array of sparks in the dark) was misperceived when the lines were looked at directly with the eyes in a secondary or tertiary position of gaze. This situation is quite different from the perception of the Helmholtz chessboard figure where the eyes are typically in the primary position and the curved lines are seen in peripheral vision. Indeed, Helmholtz noted that the curved contours of his chessboard figure were seen as curved when looked at directly. Somewhat dismissively he wrote "But, of course, the curvature of the outer rows can be perceived by looking out toward them" (Helmholtz 1910/1962, page 181). It is still not clear why Küster's and Helmholtz's observations differ.

Oomes et al (2008) have confirmed Helmholtz's observation that the illusory distortion seen with Helmholtz's chessboard pattern or isolated lines was much reduced when observers were allowed to let their gaze wander. However, when the eyes fixated the centre of the figure, in the way that Helmholtz suggested, they found that the magnitude of the illusory distortion was only about half of the value identified by Helmholtz.

Our objective in experiment 1 was also to measure the amount of systematic bias in judgments of the apparent straightness of the peripherally viewed contours of the Helmholtz chessboard pattern using three different experimental procedures. Experiment 2 was designed to investigate whether the errors in judging the straightness of lines in peripheral vision depend on the eccentricity of the lines, while in experiment 3 we looked at whether the presence and regularity of the chessboard squares play any role in the effect.

\section{Experiment 1}

\subsection{Methods}

The purpose of experiment 1 was to determine the magnitude of apparent distortion seen when viewing a wide-angle chessboard pattern similar to that used by Helmholtz (figure 5a). There are several different ways in which this can be done. In experiment 1a, we mimicked Helmholtz's procedure in which the observer was asked to approach 
towards and recede from the distorted chessboard until the pattern appeared to look like a regular chessboard pattern with straight and parallel contours (Helmholtz 1910/ 1962, page 181). Note that, as the observer approaches the pattern, any particular curved contour will stimulate more and more peripheral regions of the retina, or equivalently, the contours stimulating any particular eccentric region of the retina become less and less curved. To do this systematically, we created an electronic version of Helmholtz's figure in which the contours between the black and white checks corresponded exactly to the mathematically correct direction circles when viewed from a critical viewing distance of 0.47 times the diameter of the figure. (The viewing distance indicated under Helmholtz's figure $[A]$ is exactly 0.47 times its diameter.) In our experiments, the images were always projected onto screens at a distance of $57 \mathrm{~cm}$, which meant that the contours of the figure would project to Helmholtz's direction circles when the diameter of the projected pattern was precisely $122 \mathrm{~cm}$. Observers were able to step through a series of images of the same chessboard figure. The difference between the images was in the degree of magnification or minification with respect to the (reference) $122 \mathrm{~cm}$ diameter. In this way, the images mimicked approaching towards or receding from the chessboard figure from a distance greater than or less than the critical viewing distance of $57 \mathrm{~cm}$.

In experiment 1a, observers viewed the chessboard pattern stereoscopically and appropriate disparities were introduced into each of the stereoscopic pairs to mimic viewing the pattern from different distances. This was achieved by displacing each of the stereoscopic half-images laterally (in opposite directions) on the frontal screen so that the vergence demand of the fused pattern was increased or decreased by a particular amount. ${ }^{(4)}$ As the observers stepped through the sequence of images, they viewed a chessboard pattern that increased in size on the screens and simultaneously became more disparate in a convergent direction. As a consequence, the pattern appeared to be of more-or-less constant size as it moved closer toward the observer (or farther away), just as it would have done in Helmholtz's original demonstration. Observers were asked to select the pattern in the sequence that appeared to have straight and parallel contours. They were encouraged to bracket their chosen pattern by making sure that the neighbouring patterns in the sequence that were smaller (and depicted as farther away) had pin-cushion-shaped peripheral contours and the neighbouring patterns that were larger (and depicted as closer) had barrel-shaped peripheral contours.

In experiment $1 b$, observers were presented with a similar sequence of stereoscopic images except that the disparities between each of the stereoscopic pairs remained fixed and correct for the $57 \mathrm{~cm}$ distance of the screens. Under these conditions, observers saw the distorted chessboard pattern increase or decrease in size, but it remained at a fixed vergence distance of $57 \mathrm{~cm}$. Note that the monocular images in this sequence were identical to those presented in experiment la, apart from the small differences in perspective between the two eyes that would result from viewing a chessboard pattern binocularly at distances other than $57 \mathrm{~cm}$. The observer's task was again to select, from the sequence of 30 images, the pattern that appeared to have straight and parallel contours.

The obvious problem in Helmholtz's original procedure and in our experiments 1a and $1 \mathrm{~b}$ is that the distorted chessboard pattern changes in angular size as the observer steps through the sequence of images, so that any particular contour of the pattern becomes more or less eccentric. Helmholtz designed the figure so that, at the critical viewing distance, the contours between the black and white checks were separated by $10 \mathrm{deg}$ when measured along the central horizontal and vertical lines (Helmholtz 1910/ 1962, page 181). This meant that at viewing distances greater than the critical viewing distance the contours would be separated by less than $10 \mathrm{deg}$ (when measured along

(4) Note that these displacements produced both the correct vergence demand and the correct pattern of vertical disparities for viewing a frontal surface at different distances (Rogers and Bradshaw 1995). 
the central horizontal and vertical lines), and at viewing distances less than the critical viewing distance the contours would be separated by more than $10 \mathrm{deg}$. This characteristic was replicated in our experiments $1 \mathrm{a}$ and $1 \mathrm{~b}$ where we changed the size of the projected pattern. In experiment 1c, we kept the separation of the contours constant at $10 \mathrm{deg}$ (measured along the central horizontal and vertical lines) and, instead of varying the size of the pattern, we varied the curvature of the contours. At one end of the sequence of images the contours were physically straight and parallel (but note that they were perceived to have a convex, barrel distortion), and at the other end of the sequence the contours had a curvature that was double that of the original Helmholtz figure (and were perceived to have a concave, pin-cushion distortion). The observer's task was again to select the pattern from the sequence of images that appeared to have straight and parallel contours. The contours of the chessboard pattern were always hyperbolae (like Helmholtz's figure) with linearly increasing curvature as a function of eccentricity. Viewing was again binocular at $57 \mathrm{~cm}$ and with 0 on-screen disparities between the left and right images.

\subsection{Apparatus and procedure}

Four observers, including the two authors, took part in the experiment. Observers sat in a large-field stereoscope (Rogers and Bradshaw 1995) in front of a pair of mirrors oriented at $\pm 45^{\circ}$ in order to independently view two rear-projected $1024 \times 768$ pixel images on screens $57 \mathrm{~cm}$ from the observer's eyes (figure 6). The visible size of the projected images was limited by a circular aperture of $85 \mathrm{~cm}$ diameter corresponding to $73 \mathrm{deg}( \pm 36.5 \mathrm{deg})$ of visual angle. The images were created with Matlab and Adobe Photoshop and they were arranged as an ordered sequence of 30 images that could be stepped through by the observer using the up-down cursor keys. The starting point in the sequence of images was randomised for each new trial. Observers were asked to fixate the centre of the pattern and select the pattern in the sequence in which the contours of the chessboard pattern appeared to be straight and parallel. Good fixation was essential since, as Helmholtz noted, as soon as the eccentric, curved contours of his chessboard are directly fixated, their curvature becomes apparent.

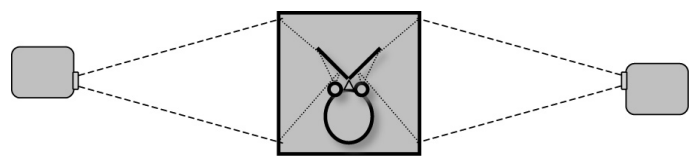

Figure 6. A plan view of the apparatus showing how the binocular images were projected from two identical LCD projectors onto screens positioned at a distance of $57 \mathrm{~cm}$ on either side of the observer. The observer viewed the binocular images via two mirrors at $\pm 45^{\circ}$ to the line of sight.

In preliminary experiments, we found that many naive observers were unable to maintain fixation throughout the duration of each trial and that, because they had an occasional glimpse of the curved, peripheral contours, their setting showed less of an effect. As a consequence, their results were significantly less consistent than those of more experienced observers. In the end, data were collected for four well-trained observers, two of whom were naive to the purpose of the experiment. In addition, we sometimes found that the most eccentric contours of the pattern still appeared to be curved when the least eccentric contour appeared to be straight (or vice versa) and, under these conditions, observers were asked to choose the best compromise for all the visible contours. Helmholtz reported a similar effect. He wrote:

"On the other hand, as to the remoter vertical rows, particularly those on the outer side of the eye, I was disposed to select a position somewhat nearer the figure. At the distance of $20 \mathrm{~cm}$, for which the pattern was designed, their actual curvature did not seem to disappear entirely." (Helmholtz 1910/1962, page 182) 


\subsection{Results}

The results for all conditions and for the four observers were normalised by expressing the magnitude of the distortion effect as a percentage of the distortion predicted by Helmholtz on the basis of his direction circles (Rogers 2008). That is, if the observer chose a particular size of pattern (in experiments 1a and 1b) or a particular level of distortion (experiment 1c) that had exactly the same level of distortion as the original Helmholtz figure at the critical viewing distance, this was designated to be a $100 \%$ effect. On the other hand, if an observer chose a chessboard pattern that had physically straight and parallel contours, this was designated to be a $0 \%$ effect.

The results of experiment la (figure 7a) show that the magnitude of the perceived distortion of the chessboard pattern in our experiment $(101 \%)$ was almost identical to that reported by Helmholtz (designated as $100 \%$ ). This conclusion was derived from the fact that the chessboard pattern that our observers selected as having straight and parallel contours was slightly smaller in angular size $(121 \mathrm{~cm}$ instead of $122 \mathrm{~cm}$ diameter) and was depicted (through disparities) at a marginally greater distance $(57.6 \mathrm{~cm}$ rather than $57 \mathrm{~cm}$ ), compared with Helmholtz's observations.

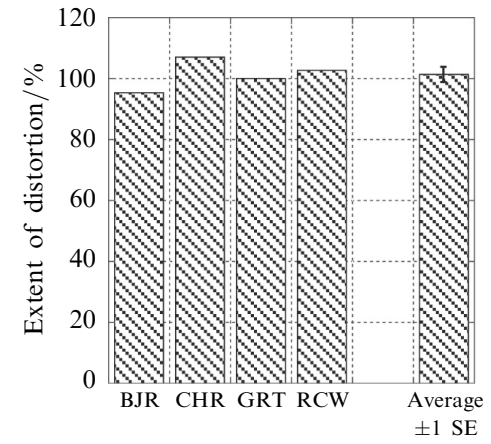

(a)

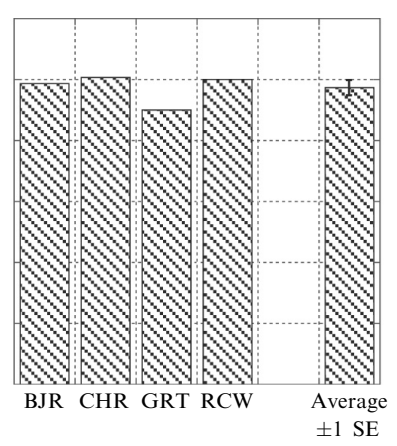

(b)

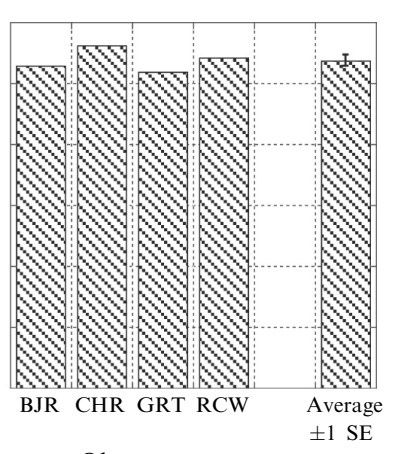

(c)

Figure 7. The bar graphs show the extent of the chessboard pattern's distortion needed for the pattern to be perceived as having straight and parallel contours. (a) The results of experiment 1a when the stereoscopic images increased in both size and crossed disparity as the observer stepped through the sequence of images. (b) The results of experiment $1 \mathrm{~b}$ when the stereoscopic images increased in size but the disparity between the stereoscopic images was kept constant and appropriate to the $57 \mathrm{~cm}$ viewing distance. (c) The results of experiment $1 \mathrm{c}$ when the separation of the contours was kept constant at $10 \mathrm{deg}$ and the degree of curvature of the contours was varied. Note that the images that correspond to a ' $100 \%$ ' effect were identical in all three conditions.

Since the pattern subtended a slightly smaller visual angle for the contours to appear straight and parallel in our experiments, those contours were actually more curved (for a given eccentricity) than at Helmholtz's critical distance and hence the magnitude of the effect was marginally larger than that reported by Helmholtz. In experiment $1 b$, the observer was again asked to choose the figure whose contours appeared straight and parallel but, unlike experiment 1a, the binocularly viewed figure was always presented with disparities appropriate for $57 \mathrm{~cm}$. In this case, the results show that the Helmholtz figure needed to subtend almost exactly the same visual angle as that predicted on the basis of Helmholtz's direction circles for the contours to appear straight and parallel. The average magnitude of the effect under these conditions was $97 \%$. In experiment 1c, the contours between the checks were always separated by $10 \mathrm{deg}$ (along the central horizontal and vertical axes), as in the original Helmholtz figure. However, the sequence of images from which the observer was asked to choose the one whose contours appeared straight and parallel contained a set of images in which the contours of the figure were curved to a greater or lesser extent. Under these conditions, the average magnitude of the effect was found to be $107 \%$. 
Informally, several of the observers also reported that when they had selected a particular image as being the one in which the contours separating the chessboard squares appeared to be straight and parallel, they perceived the surface to be slightly concave rather than flat. Helmholtz noted a similar effect. He wrote:

"Thus, when I let my eye wander, I see the drawing curved like a flat bowl, the curvature of the hyperbolas seeming to be a curvature of the surface, and the lines in this curved surface being apparently great circles (or shortest lines)." (Helmholtz 1910/1962, page 181)

We found this concave, bowl-like appearance to be significantly enhanced when the chessboard pattern was viewed monocularly, and therefore in the absence of the disparities specifying the pattern to be flat and in a frontal plane. We shall return to this point later in the discussion.

\subsection{Summary of the results from experiment 1}

The results of all three conditions of experiment 1 show that when the original (pincushion) chessboard figure was presented at the critical viewing distance for the contours to be mapped onto Helmholtz's direction circles $(122 \mathrm{~cm}$ diameter at $57 \mathrm{~cm})$, the contours appeared to be approximately straight and parallel. Moreover, the magnitude of the distorted chessboard effect was similar under the three different viewing conditions and is very similar to Helmholtz's original estimate. As a consequence, our conclusion is the same as Helmholtz's - straight contours in peripheral vision are perceived as curved concave towards the fixation point and contours with a significant convex curvature towards the fixation point (ie pin-cushioned) are perceived as straight when seen in peripheral vision.

\section{Experiment 2}

Both Helmholtz's original demonstration with the distorted chessboard figure and our own experiments clearly demonstrate that our perception of the pin-cushioned figure can be significantly in error. However, although the magnitude of the perceptual errors is striking, the use of the chessboard figure does not allow us to test Helmholtz's direction-circle hypothesis adequately or determine the factors that contribute to the illusion. For example, it is not clear whether the extent of illusory distortion of the peripherally viewed contours is the same for all eccentricities, and it is not clear whether the presence of chessboard squares affects our perception of the straightness of the contours separating those squares. In order to address the first question, we created three modified versions of the chessboard figure in which there were just isolated hyperbolic contours with no chessboard squares (figure 8). In the first version, the hyperbolic contours (two vertical and two horizontal) were located \pm 10 deg to either side of the fixation point so that their locations corresponded to the innermost contours of the original

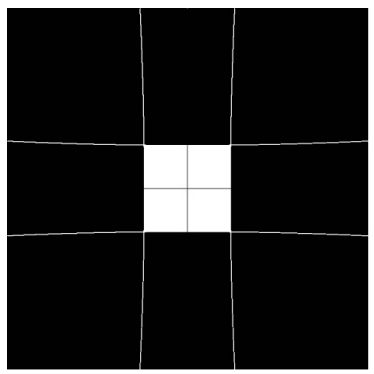

(a)

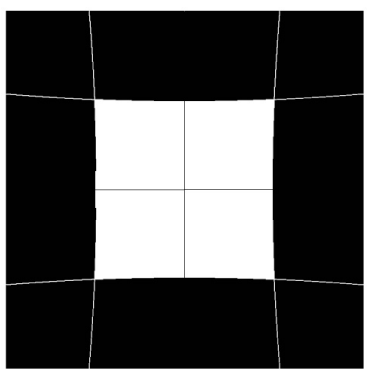

(b)

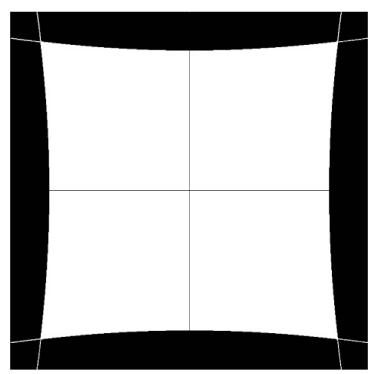

(c)

Figure 8. The three different stimulus patterns used in experiment 2 . The contours were located at (a) $\pm 10 \mathrm{deg}$, (b) $\pm 20 \mathrm{deg}$, or (c) $\pm 30 \mathrm{deg}$ (measured along the central horizontal and vertical lines) on either side of the fixation point. 
Helmholtz figure. In the second version, the contours were located \pm 20 deg to either side of the fixation point (corresponding to the second set of peripheral contours in the chessboard figure), and in the third, they were \pm 30 deg to either side of the fixation point.

In this way, we could test whether the extent of the distortion predicted by Helmholtz on the basis of his direction circles was the same or different for different eccentricities. The same large-field stereoscope was used to display the images as in experiment 1 . Viewing was binocular and the disparities were appropriate for the $57 \mathrm{~cm}$ viewing distance as in experiment 1c. The images were again created with Matlab and Adobe Photoshop and arranged as a sequence of 30 images that could be stepped through by the observer with the up-down cursor keys. The range of curvatures of the contours in the sequence of images was the same as in experiment 1c. The starting point in the sequence of images was randomised for each new trial. Observers were asked to fixate the centre of the pattern and select the particular image in the 30-image sequence in which the contours of the pattern appeared to be straight and parallel.

\subsection{Results}

The results for the three conditions and for the four observers were normalised by expressing the magnitude of the distortion effect as a percentage of the distortion predicted by Helmholtz on the basis of his direction circles. That is, if the observer chose a particular level of distortion that had exactly the same level of distortion as the original Helmholtz figure at the critical viewing distance, this was designated to be a $100 \%$ effect. On the other hand, if an observer chose a pattern that had physically straight and parallel contours, this was designated to be a $0 \%$ effect.

The results of experiment 1 confirmed Helmholtz's finding that when the contours of the chessboard pattern appear to be straight and parallel, those contours lie roughly along his direction circles. The results of experiment 2 present a rather different picture.

First, when pairs of isolated contours are used rather than the chessboard pattern, observers judged the contours to be straight and parallel when they had much less curvature than that predicted by the direction-circles hypothesis. Typically, the isolated contours had to have only $49 \%-90 \%$ of the curvature of the corresponding contours of the chessboard figure for them to appear to be straight and parallel. Moreover, in comparison with experiment 1 , there were much larger individual differences in the settings, although the patterning of the results over the three conditions was very similar for all observers. In addition, the magnitude of the illusory effect depended crucially on the eccentricity of the contours. For the pairs of contours located $10 \mathrm{deg}$ from the fixation point (figure $9 \mathrm{a}$ ), the contours had to have, on average, $90 \%$ of the

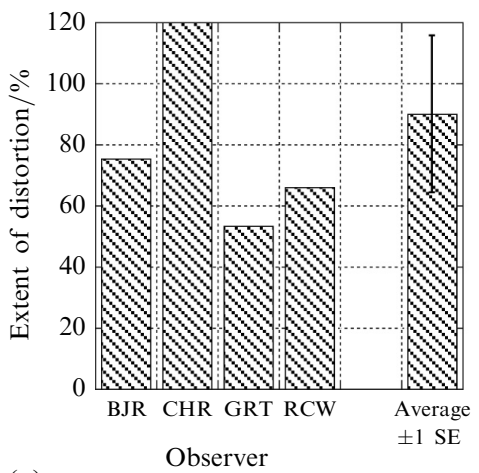

(a)

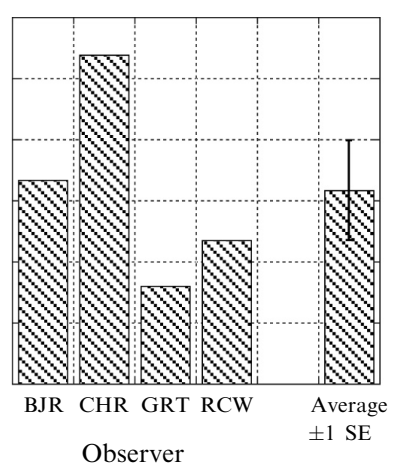

(b)

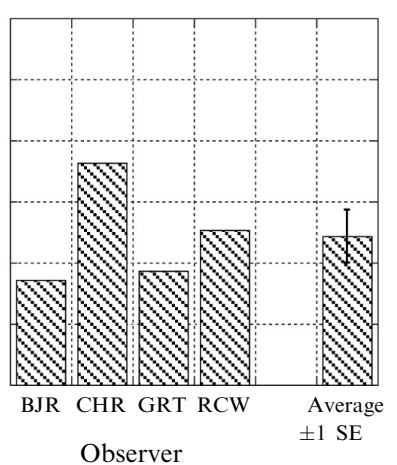

(c)

Figure 9. The bar graphs show the extent of the pattern's distortion needed for the contours to be perceived as straight and parallel. (a) The results with the pairs of contours $10 \mathrm{deg}$ on either side of the fixation point. (b) and (c) The results for contours with 20 deg and 30 deg of eccentricity, respectively. 
curvature of the corresponding contours of the chessboard figure. Contours located $20 \mathrm{deg}$ from the fixation point (figure $9 \mathrm{~b}$ ) had to have $\sim 63 \%$ of the curvature of the corresponding contours of the chessboard figure, and those located $30 \mathrm{deg}$ from the fixation point, only $\sim 49 \%$. Why should this be?

Helmholtz also noted that the extent of the distortion seen with the chessboard figure decreased with increasing eccentricity. He wrote:

\footnotetext{
"On the other hand, as to the remoter vertical rows, particularly those on the outer side of the eye, I was disposed to select a position somewhat nearer the figure [i.e. with less curved contours]. At the distance of $20 \mathrm{~cm}$, for which the pattern was designed, their actual curvature did not seem to disappear entirely." (Helmholtz 1910/1962, page 183)
}

Our own results and Helmholtz's observations are consistent with the possibility that observers relied primarily on the perceived curvature of the innermost contours when making their judgments with the chessboard figure but this factor alone cannot account for the fact that the extent of the illusory distortion in experiment 2 was less than $100 \%$, even for the closest (10 deg) pairs of contours. As we indicated previously, the second factor that distinguished the isolated contour patterns used in experiment 2 from the chessboard pattern used in experiment 1 was the presence of the chessboard squares. How could the presence of the chessboard squares in experiment 1 affect the magnitude of the perceived distortion?

\section{Experiment 3}

In reporting the results for experiment 1, we noted that observers often perceived the surface on which the chessboard pattern was seen to be concave at the point at which they judged the contours to be straight and parallel. This is not surprising since a pin-cushion chessboard image on the retina could be the projection of a pin-cushion figure on a frontal surface with non-parallel, hyperbolic contours between the 'squares'; (figure $5 \mathrm{~b}$ ) or an array with parallel contours superimposed on a concave surface. ${ }^{(5)}$ If the visual system were to assume that chessboard 'squares' are generally similar in size and that the contours between them are parallel, the greater extent of the distortion found in experiment 1 compared to experiment 2 could be due to the fact that a greater amount of pin-cushion distortion is tolerated because it can be 'accounted for' by the curvature of the surface on which it is seen. Putting the argument the other way around, there would be less reason to assume that the single, isolated contours in experiment 2 were parallel, and therefore they are more likely to be seen for what they are-curved and lying on a frontal surface. To investigate this possibility, we repeated experiment 2 using the same pairs of contours at eccentricities of $\pm 20 \mathrm{deg}$ and $\pm 30 \mathrm{deg}$ as in experiment 2 but, in this experiment, the contours were filled with chessboard squares (figure 10). The apparatus and procedure were the same as in experiment 2. If our hypothesis is correct, the amount of illusory distortion should be greater for the chessboard patterns used in this experiment compared with the results obtained in experiment 2 with isolated contours.

\subsection{Results}

The results for the two conditions and the four observers were normalised by expressing the magnitude of the distortion effect as a percentage of the distortion predicted by Helmholtz on the basis of his direction circles, as in experiments 1 and 2 (figure 11).

(5) If 'vertical' and 'horizontal' lines of latitude are marked on a spherical concave surface, these will be parallel and equally spaced (by definition) as shown in figure 4b. Note that, although the contours between the 'squares' are parallel in this situation, the squares themselves become very distorted towards the far corners. 


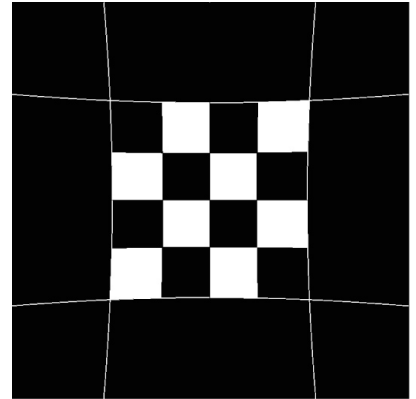

(a)

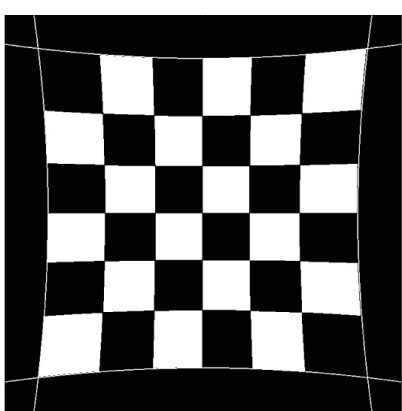

(b)

Figure 10. The two different stimulus patterns used in experiment 3. The contours were located (a) \pm 20 deg or (b) \pm 30 deg (measured along the central horizontal and vertical lines) on either side of the fixation point, and the area between the contours was filled with the same chessboard squares as used in experiment 1.

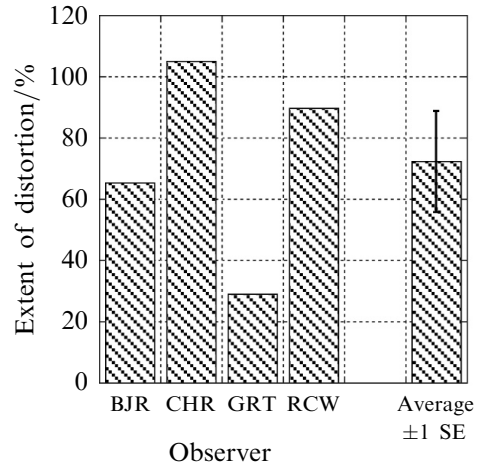

(a)

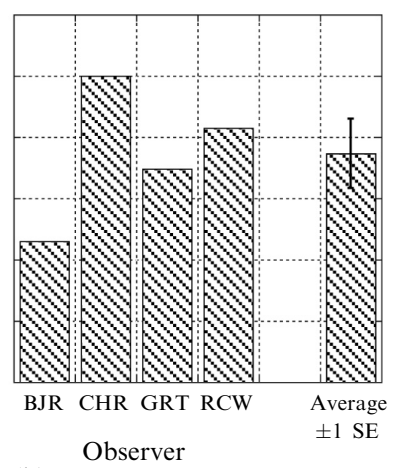

(b)

Figure 11. The bar graphs show the extent of the pattern's distortion needed for the contours to be perceived as straight and parallel. (a) The results with the pairs of contours \pm 20 deg on either side of the fixation point and (b) the results for contours with a \pm 30 deg of eccentricity.

The amount of distortion perceived in the $\pm 20 \mathrm{deg}$ outline contours when filled with chessboard squares was equivalent to $\sim 72 \%$ of the distortion predicted by the direction-circle hypothesis, and was significantly greater than that found for the outline contours alone $(\sim 63 \%)$. The result is consistent with our suggestion that a greater amount of pin-cushion distortion is tolerated when the chessboard squares are present because it can be 'accounted for' by the curvature of the surface on which it is seen. The magnitude of the effect for the $\pm 20 \mathrm{deg}$ outline contours filled with chessboard squares $(\sim 75 \%)$, was also larger than that found for the outline contours alone $(\sim 49 \%)$. Both these results are consistent with our hypothesis that the visual system is more likely to assume that the contours are parallel when (i) there are more of them and (ii) they are filled with chessboard squares, than for the single, outline contour condition. The pattern of results is also consistent with the observation that the chessboard patterns used in this experiment were more often seen as covering a concave surface than was true for the outline contours of experiment 2.

\section{Summary and discussion}

The results of all three experiments confirm Helmholtz's finding that the 'uncurved lines' on the visual globe - that is, the peripheral lines that appear to be straight - have to be curved convex towards the fixation point. Expressed another way, straight lines seen in peripheral vision typically appear to be curved concave towards the fixation point. 
Although the direction of the distortion in our experiments is consistent with Helmholtz's observations, the magnitude of the effect was found to depend on the particular characteristics of the display. When Helmholtz's chessboard pattern was used, the boundary contours between the black and white checks were seen as straight when they had $\sim 100 \%$ of the curvature predicted by Helmholtz's direction-circles hypothesis. On the other hand, when a single 'square' contour was used in place of the chessboard pattern, the extent of the distortion was greatly reduced.

Helmholtz observed (and we have confirmed) that the distorted chessboard figure is often seen to have a concave, bowl-like 3-D shape. This is particularly evident when the figure is viewed monocularly and the observer makes head movements towards and away from the figure, as the reader can verify for himself or herself. The fact that the distorted figure is seen as concave is not surprising - the only surface covered with parallel and equally spaced contours that creates an image identical to the Helmholtz chessboard figure with diverging contours is one that has a concave 3-D profile (footnote 4 and figure $4 \mathrm{~b}$ ). Moreover, there is little contradictory information that the surface on which the contours are drawn is actually flat when the figure is viewed monocularly, which is in contrast to the stereoscopic viewing situation used in our experiments.

We have also noticed that, when approaching the distorted chessboard figure with only one eye open, the contours between the squares stay more-or-less straight and parallel, but the 3-D shape of the surface on which they are seen becomes less concave. On the other hand, when the same figure is viewed binocularly, so that there is disparity information to specify that the surface is actually flat, observers reported significantly less concavity in the surface shape and that 3-D appearance changed by a smaller amount as she or he approached the figure.

Consistent with these observations, we have also found that, when Helmholtz's chessboard figure is viewed binocularly from a distance greater than the critical value, the apparent concavity of the 3-D surface can be nulled out, or even reversed, by introducing a convex pattern of disparities across the figure. However, although the disparities across the centre of the figure do make it appear convex, the surface in the outer annular region (with an eccentricity of $>20$ deg from fixation) retains its apparent concave shape. This suggests that the pattern of convex disparities is only effective in influencing our perception of the surface shape within an area of around $40 \mathrm{deg}$ diameter ( $\pm 20 \mathrm{deg}$ of the fixation point) and that the visual system is either less sensitive or totally unable to use horizontal disparities beyond this limit (eg Prince and Rogers 1998).

So how should we answer Helmholtz's question: "Which are the uncurved lines on the visual globe"? It is clear that our perception of the straightness of long lines in peripheral vision is not veridical, and that peripheral lines have to be curved convex towards the fixation point for them to appear straight, as Helmholtz originally observed. However, it would appear that the lines that are seen as straight in peripheral vision do not necessarily correspond to Helmholtz's 'direction circles', and the extent of the distortion illusion seems to depend critically on the particular experimental conditions. Moreover, it is not clear from a geometrical standpoint that there could be any single answer to Helmholtz's question. We have pointed out previously that, while it is true that straight lines in the surrounding world project to great circles on a spherical projection surface (figure 1), there are lines in world with 3-D curvature that also project to great circles (Rogers and Brecher 2007). Any curved arc that has its centre of curvature at the eye is an example of this fact. Thus, although the stimulation of a great circle on a spherical projection surface provides potential information about the 2-D alignment of points from a particular vantage point, such stimulation cannot, in principle, tell us about the straightness of a line in 3-D space, since this 
requires additional information about the 3-D profile of the line or the relative distances of the distal points. The following example makes this clear. Helmholtz's three stars in the sky may be collinear from the position of the Earth (verified by holding up a stretched string to 'connect' the three stars) and, as a consequence, they will all stimulate the same arc of a great circle on a spherical projection surface (figure 2), but it would be very unlikely that those three stars are actually aligned and could be connected by a straight line through space! Thus it would seem that, in principle, there can be no 'uncurved' loci that could inform us about the straightness of a line in space.

There is an additional problem. Helmholtz asked "Which are the uncurved lines on the visual globe?" Our geometrical analysis (figure 1) shows that straight lines in the world map onto great circles on the field of fixation (or celestial sphere), but the location of those lines on the visual globe (or the retina) depends on eye position. Hence, unless there is additional information about eye position, the visual system would not be able to distinguish between straight lines seen from one eye position and curved lines seem from a different eye position.

A less ambiguous (but perhaps more meaningful) task than the determination of whether a single peripheral line in the world is straight would be to ask whether two lines are parallel. Indeed, the judgment of the extent of distortion in the Helmholtz chessboard figure is probably based, at least in part, on whether the boundary contours between the checks appear to be parallel. The same problem arises, however. Two lines that are parallel on a frontal surface (figure 1) will stimulate a pair of great circles on a spherical projection surface. Two lines that are also parallel but lie on an equidistant surface (figure 2) will stimulate a pair of lines of latitude on a spherical projection surface. This shows that there can be no way of determining whether two lines are parallel from their location in the visual field without additional information about the 3-D profile of the surface on which the two lines are drawn. Hence, it is possible that the reason why the contours of the Helmholtz chessboard figure are misperceived is because there is insufficient information about the 3-D profile of the surface of the figure. This would be particularly problematic with monocular viewing and in far peripheral vision. Could the reason why Helmholtz and others have found that the contours of his figure appear to be straight when they stimulate locations in-between the great circles and lines of latitude (figure 5) is not because they stimulate some special compromise loci such as 'direction circles' but rather because 3-D shape of the surface of the figure is assumed to lie somewhere between a frontal surface and an equidistant surface, in the absence of sufficient 3-D information?

\section{Experiment 4}

The preceding analysis shows that it is impossible to judge whether a pair of lines is parallel on the basis of the retinal loci alone-we need additional information about the distance to the lines or, equivalently, the shape of the surface on which the lines lie. How good are we at using this additional information in practice? To answer this question we carried out a fourth experiment in which observers were asked to judge when two eccentrically positioned close-to-horizontal contours appeared to be parallel under two different viewing conditions. In the first condition, the contours were located on a disparity-defined, frontal surface (figure 12a) and, in the second, the contours were curved around a disparity-defined and equidistant cylindrical surface (figure 12b).

Note that in the first case, contours that are physically parallel project to great circles while in the second case they project to lines of latitude. In both cases, the two contours were located $10 \mathrm{deg}$ above and below the fixation point (measured along the central vertical line) and the area between the two contours was filled with a random texture in order to provide maximal disparity information about the surface (frontal or 


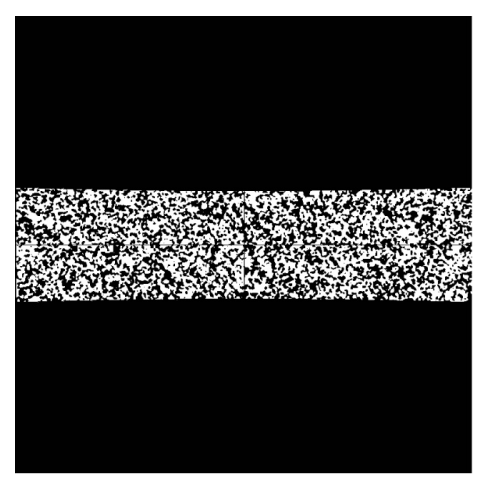

(a)

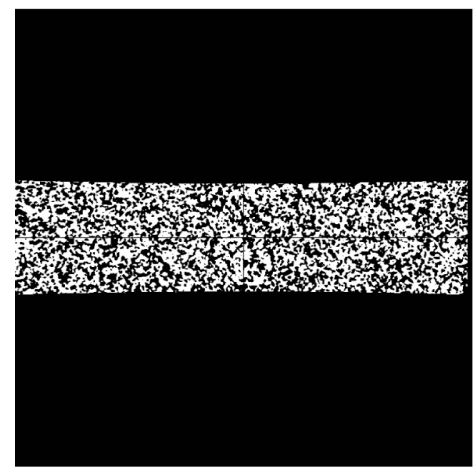

(b)
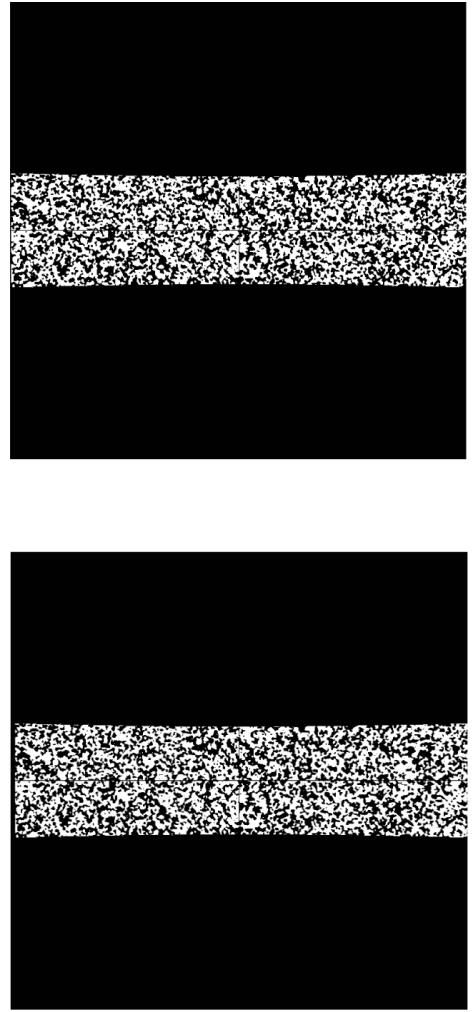

Figure 12. Stereopairs used in experiment 4, suitable for cross-eye fusion. The bounding contours of random texture are identical in (a) and (b) but the disparities between the texture elements in (a) specify a frontal surface whereas those in (b) specify an equidistant surface (when viewed under experimental conditions). With fixation at the centre of the patterns, the bounding contours of (a) appear to diverge with increasing eccentricity whereas the bounding contours of (b) appear to converge (in the actual experiment).

cylindrical) between the bounding contours. The locations of the contours were identical to the \pm 10 deg 'horizontal' contours shown in figure $8 \mathrm{a}$.

The observer's task was to adjust the curvature of those contours until they appeared to be parallel. Viewing was binocular with disparities appropriate to a frontal surface $57 \mathrm{~cm}$ in front of the observer, in the first case, and to a vertical, cylindrical surface with a radius of $57 \mathrm{~cm}$ from the observer's cyclopean point, in the second case. To introduce disparities appropriate to an equidistant, cylindrical surface into the image pairs we used Adobe Photoshop with linearised, sub-pixel interpolation. The curvature of the bounding hyperbolic contours $10 \mathrm{deg}$ above and below the fixation point was varied in the same way as in experiments $1 \mathrm{c}$ and 2 to create a 30 -image sequence. Observers were asked to fixate the centre of the pattern and select, with the up-down cursor keys, the particular image in which the two contours of the pattern appeared to be straight and parallel. Note that the monocular images of the bounding contours for the two conditions (frontal surface and cylindrical surface) were the same-only the disparity profile of the texture between those contours was different.

\subsection{Results}

The results for the two conditions and for the four observers were normalised by expressing the magnitude of the distortion effect as a percentage of the distortion predicted by Helmholtz on the basis of his direction circles, as in the previous experiments. If observers 
based their judgments on the local of Helmholtz's direction circles, the results should be close to $100 \%$. However, if observers were able to use optimally the information about the shape of the surface on which the contours were projected, the results should be close to $0 \%$ for the frontal surface condition and close to $200 \%$ for the equidistant cylindrical surface condition (since Helmholtz's direction circles lie halfway between the location of great circles and the lines of latitude on a spherical projection surface-figure $5 \mathrm{~b}$ ).

The results show that observers do not perform veridically when asked to make a judgment about whether two lines on a frontal surface are parallel (figure 13a). Instead, they choose contours that have a convex curvature with respect to the fixation point, as Helmholtz first reported. However, the extent of the curvature $(\sim 68 \%)$ is significantly less than that predicted by the direction-circles hypothesis $(100 \%)$. The magnitude of the effect is also significantly less than the results we described previously for $\pm 10 \mathrm{deg}$ isolated contours used in experiment $2(\sim 90 \%)$. The most likely explanation for the difference is that the presence of the background texture in this final experiment was much more effective in 'locking' the contours to the frontal plane than the isolated contours used in experiment 2

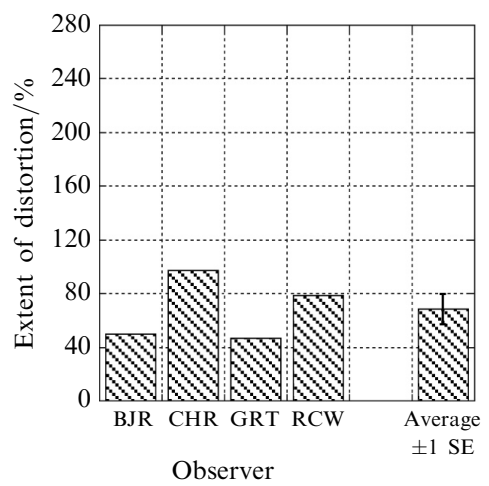

(a)

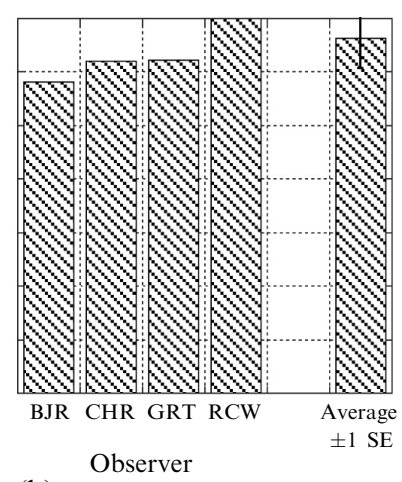

(b)

Figure 13. The bar graphs show the extent of the distortion (as percentage of the Helmholtz figure) needed for the contours to be perceived as straight and parallel: the results for a pair of $\pm 10 \mathrm{deg}$ 'horizontal' contours superimposed on a frontal surface (a) and the results for a pair of contours superimposed on an equidistant surface (b). Note the difference in scale of the ordinate in this graph compared to the previous graphs.

This was confirmed by observers' reports that the contours and texture in the frontal viewing condition of the present experiment always appeared to lie close to a frontal plane, whereas in experiment 2 observers frequently remarked on the concave bowl-shaped appearance of the figure.

The results for exactly the same contours superimposed on an equidistant surface are very different (figure 13b). Observers chose contours with a very substantial convex curvature that was actually greater than the curvature that would stimulate lines of latitude on a spherical projection surface, ie twice as great as the curvature of Helmholtz's chessboard pattern based on direction circles (figure $3 b$ ). Together, these two results are important because they show that with identical sets of images (apart from the disparity differences between the texture elements in the left and right views), observers choose very differently shaped contours when asked to judge which contours appear to be straight and parallel. Moreover, the results reinforce the theoretical point that there can be no 'uncurved lines on the visual globe' and that observers' judgments about the straightness and parallelness of contours depend crucially on information about the distance to the contours or, equivalently, the shape of the surface upon which the contours are superimposed. 


\section{Conclusions}

We have confirmed Helmholtz's observation that the perception of peripherally viewed lines can be non-veridical-physically straight lines can appear curved, concave towards the fixation point, and lines curved convex towards the fixation point can appear to be straight. In addition, we have quantified the magnitude of the effect for the first time under a wide variety of different viewing conditions. Our results show that the magnitude of the apparent distortion depends critically on the particular viewing conditions. This suggests that there are no particular 'uncurved lines on the visual globe' and, moreover, there does not appear to be any special significance to Helmholtz's 'direction circles'. Theoretical considerations (rather than empirical evidence) point to the fact that there could not, in principle, be any loci on the visual globe (or on our retinae) that could reliably inform us whether a given line is straight or a pair of lines is parallel because both judgments also require information about the 3-D properties of the surface on which the line or lines lie. According to this analysis, the misperception of the contours of the Helmholtz figure can be seen as a result of the paucity of 3-D information in peripheral vision and the use of a regular texture like the chessboard pattern, rather than as a consequence of using a strategy based on a particular set of 'uncurved lines on the visual globe'.

Acknowledgment. Cassandra Rogers was supported by a Networking Grant from the McDonnell Foundation.

\section{References}

Anstis S M, 1998 "Picturing peripheral acuity" Perception $27817-825$

Gibson J J, 1966 The Senses Considered as Perceptual Systems (Boston: Houghton-Mifflin)

Hansen R, 1973 "This curving world" Journal of Aesthetic and Art Criticism 32 147-161

Helmholtz H von, 1867 Handbuch der physiologischen Optik 1st edition, volume 9 of Allgemeine Encyclopädie der Physik Ed. G Karsten (Leipzig: Voss)

Helmholtz H von, 1910/1962 Treatise on Physiological Optics English translation by J P C Southall for the Optical Society of America (1925) from the 3rd German edition of Handbuch der physiologischen Optik (Hamburg: Voss, 1910) (New York: Dover, 1962)

Howard I P, Rogers B J, 1995 Binocular Vision and Stereopsis (New York: Oxford University Press)

Küster F 1876 "Die Direktionskreise des Blickfeldes" Archiv für Ophthalmologie 22 149-210

Liu L, Schor C M, 1998 "Functional division of the retina and binocular correspondence" Journal of the Optical Society of America A $151740-1755$

Oomes A H J, Koenderink J J, Doorn A J van, Ridder H de, 2008 "Helmholtz's checkerboard reviewed" Perception 37 Supplement, 115

Parlee M B, 1972 "Differences in apparent straightness of dot and line stimuli" Vision Research 12 $735-741$

Pirenne M H, 1970 Optics, Painting and Photography (London: Cambridge University Press)

Prince S J D, Rogers B J, 1998 "Sensitivity to disparity corrugations in peripheral vision" Vision Research $382533-2537$

Rogers B J, 2008 "Helmholtz's celestial sphere and the perception of straight lines" Perception 37 Supplement, 125

Rogers B J, Bradshaw M F, 1995 "Disparity scaling and the perception of frontoparallel surfaces" Perception $24155-179$

Rogers B J, Brecher K, 2007 “Straight lines, 'uncurved lines', and Helmholtz's 'great circles on the celestial sphere" " Perception $361275-1289$

Simonsz H J, Den Tonkelaar I, 1990 "19th Century mechanical models of eye movements, Donders' law, Listing's law and Helmholtz' direction circles” Documenta Ophthalmologica 74 95-112 


\section{PERTEPTION}

VOLUME 382009

www.perceptionweb.com

Conditions of use. This article may be downloaded from the Perception website for personal research by members of subscribing organisations. Authors are entitled to distribute their own article (in printed form or by e-mail) to up to 50 people. This PDF may not be placed on any website (or other online distribution system) without permission of the publisher. 